\title{
¿EL EMBARAZO EN ADOLESCENTES ES SIEMPRE DE ALTO RIESGO?
}

\section{Dr. Alberto Duarte-Contreras, M.D. 洗}

En la literatura universal sobre embarazo en adolescentes encontramos diversidad de datos, contradictorios en muchas ocasiones en lo que se relaciona a complicaciones del embarazo y del parto, a trabajo de parto, a parto prematuro, a bajo peso de productos, a morbimortalidad materno fetal, a malformaciones congénitas, en forma tal que hacen enigmático este capítulo, más cuando no se ha tenido un concepto definido sobre la edad límite para su estudio. Y entre más trabajos se publican, más enigmáticos se tornan aún algunos de sus problemas. Nos propusimos investigar si el factor edad de por sí es el determinante de ciertas complicaciones del embarazo en adolescentes, o si, por el contrario, como en toda gestante, hay otras causas o circunstancias especiales que comprometen la integridad de esta madre y la de su hijo.

Sabemos que es muy complejo poner en edad límite para el estudio de problemas de adolescencia, pero hemos escogido los 16 años para seguir la orientación que al respecto dá Hauffman (17).

\section{Material y Métodos}

Tomamos como material de estudio 1.335 Historias Clínicas de adolescentes de 11 a 16 años atendidas en la Maternidad del Hospital San Juan de Dios, Cúcuta, entre el 1\% de Enero de 1960 y el 31 de Diciembre de 1974, todas de bajo nivel socioeconómico. Las dividimos en dos grupos: Grupo A de 11 a 15 años y Grupo B de 16 años. Comparamos los datos obtenidos con el grupo de pacientes de mayor edad, 17 a 44 años.

\section{Resultados}

De las 1.335 adolescentes, 668 pertenecen al Grupo A y 667 al Grupo B, lo qpe nos dá una incidencia de $1.65 \%$ para cada uno de los grupos, un embarazo por cada 66 pacientes atendidas en el Servicio. El hecho de haber igual número de pacientes en los dos Grupos facilita la comparación de los índices obtenidos.

El aumento acelerado de embarazos en adolescentes a partir de 1966 descendió de manera notoria en 1972 y 1973.

La MENARCA se presentó a los 12.4 años promedio para el Grupo A y a los 13.3 años para el Grupo B, lo que da una edad promedio de 12.8 años para el grupo total de adolescentes. El promedio del Servicio es de 13.4 años (Cuadro 2).

Las RELACIONES SEXUALES se iniciaron entre los 10 y los 15 años.

En el Grupo A hubo 479 solteras $(71.7 \%)$; en el Grupo B las encon-

* Del Departamento de Gíneco Obstetricia del Hospital San Juan de Dios. Cúcuta, Colombia. 


\section{CUADRO N: 1}

INCIDENCIA POR EDAD Y POR AÑO

\begin{tabular}{|c|c|c|c|c|c|c|c|c|c|}
\hline Año & $\begin{array}{c}\text { Toial } \\
\text { pacientes }\end{array}$ & 11 & 12 & $\begin{array}{l}\text { de las } \\
13\end{array}$ & $\begin{array}{r}\text { adoles } \\
14\end{array}$ & 15 & 16 & $\begin{array}{c}\text { Total } \\
\text { adolescentes }\end{array}$ & $\%$ \\
\hline 1960 & 1.220 & - & - & - & 3 & 4 & 12 & 19 & 1.5 \\
\hline 1961 & 1.782 & -- & - & - & 2 & 7 & 21 & 30 & 1.2 \\
\hline 1962 & 2.070 & 一 & - & - & 5 & 10 & 25 & 40 & 1.4 \\
\hline 1963 & 2.244 & - & - & - & 2 & 14 & 32 & 48 & 1.2 \\
\hline 1964 & 1.883 & - & - & 1 & - & 18 & 27 & 46 & 2.4 \\
\hline 1965 & 2.513 & $\cdots$ & - & - & 8 & 19 & 34 & 61 & 2.0 \\
\hline 1966 & 3.030 & $\cdots$ & - & 2 & 10 & 33 & 42 & 87 & 2.8 \\
\hline 1967 & 2.583 & 1 & - & 1 & 7 & 41 & 38 & 88 & 3.4 \\
\hline 1968 & 3.521 & - & - & - & 12 & 51 & 44 & 107 & 3.0 \\
\hline 1969 & 3.649 & - & 1 & 2 & 15 & 50 & 59 & 127 & 3.4 \\
\hline 1970 & 4.252 & - & 1 & 1 & 13 & 59 & 77 & 151 & 3.5 \\
\hline 1971 & 4.673 & - & - & 3 & 19 & 77 & 83 & 182 & 3.8 \\
\hline 1972 & 4.481 & 1 & 1 & 1 & 15 & 14 & 63 & 125 & 2.7 \\
\hline 1973 & 3.895 & - & - & 2 & 17 & 32 & 51 & 102 & 2.7 \\
\hline 1974 & 3.686 & - & - & 4 & 11 & 48 & 59 & 122 & 3.3 \\
\hline Total & 45.487 & 2 & 3 & 17 & 139 & 507 & 667 & 1335 & 2.9 \\
\hline
\end{tabular}

\section{CUADRO $N$ № 2}

MENARCA

\begin{tabular}{lrc} 
Edad & $\begin{array}{r}\text { Número de pacientes } \\
\text { Grupo A }\end{array}$ & \begin{tabular}{c} 
Grupo B \\
\hline 8
\end{tabular} \\
9 & 1 & - \\
10 & 2 & - \\
11 & 10 & - \\
12 & 75 & 40 \\
13 & 219 & 103 \\
14 & 207 & 190 \\
15 & 144 & 213 \\
Sin determinar & - & 105 \\
\hline
\end{tabular}

tramos en menor proporción, 359 $(53.4 \%)$. A medida que aumenta la edad de las adolescentes encontramos un mayor número de casadas (Cuadro 3 ).

\section{CUADRO NN 3}

\section{ESTADO CIVIL}

\begin{tabular}{lccccccc}
\hline Estado & \multicolumn{2}{c}{ Grupo A } & \multicolumn{2}{c}{ Grupo B } & \multicolumn{2}{c}{ Total } \\
\hline civil & $\mathbf{N}^{\mathbf{0}}$ & $\%$ & $\mathbf{N}^{\mathbf{0}}$ & $\%$ & $\mathbf{N}^{\mathbf{0}}$ & $\%$ \\
Solteras & 479 & 71.7 & 359 & 53.4 & 838 & 62.5 \\
Casadas & 189 & 28.3 & 308 & 46.6 & 497 & 37.5 \\
TOTAL & 668 & & 667 & & 1335 & \\
\hline
\end{tabular}

Asistieron por una o más veces a CONTROL PRENATAL 130 pacientes del Grupo A (19.1\%) y 287 del Grupo B $(43 \%)$. El promedio de consultas para quienes asistieron a control en el Grupo A fue de 1.7 y de 3.9 en el Grupo B. Vale la pena observar que hubo un mejor control prenatal en el Grupo B, grupo en el cual encontramos el mayor número de casadas.

El $11.9 \%$ de las adolescentes del Grupo A y el $8.8 \%$ del Grupo B terminaron su embarazo en ABORTO entre las 7 y 14 semanas, siendo más frecuente entre las semanas 12 y 14 (Cuadro 4).

\section{CUADRO N: 4}

TERMINACION DEL EMBARAZO

\begin{tabular}{lrrrrr} 
& \multicolumn{2}{c}{ Aborto } & \multicolumn{3}{c}{ Parto } \\
Edad & $N^{0}$ & $\%$ & \multicolumn{1}{c}{ No $^{2}$} & $\%$ \\
\hline Grupo A & 80 & 11.9 & 588 & 88.1 \\
Grupo B & 59 & 8.8 & 608 & 91.2 \\
TOTAL & 139 & 10.35 & 1196 & 89.65 \\
\hline
\end{tabular}

El índice de aborto para el total de adolescentes atendidas es de 10.35\%, 
cifra inferior a la del grupo de pacientes de 17 a 44 años del Servicio que es de $20.6 \%$ (cifra que incluye un elevado número de abortos provocados). Encontramos dos ABORTOS SEPTICOS en el Grupo B sin que podamos afirmar que se tratase de abortos provocados. A la mayoría de estas pacientes se les practicó legrado instrumental y estudio anatomopatológico del material extraído.

El índice de aborto es más elevado en las gestantes de menor edad como lo observamos en el Cuadro 5.

\section{CUADRO № 5}

\begin{tabular}{lcrr}
\multicolumn{4}{c}{ ABORTO } \\
\hline Eciad & $\begin{array}{c}\text { Total } \\
\text { adolescentes }\end{array}$ & No & Abortos \\
\hline 11 & 2 & 2 & 100.0 \\
12 & 3 & 2 & 66.6 \\
13 & 17 & 8 & 47.0 \\
14 & 139 & 25 & 17.9 \\
15 & 507 & 43 & 8.4 \\
16 & 667 & 59 & 8.7 \\
& 1335 & 139 & 10.35 \\
\hline
\end{tabular}

Hubo tres ABORTOS MOLARES en el Grupo B (una MOLA por cada 445 embarazos del grupo de adolescentes).

No encontramos EMBARAZO ECTO$\mathrm{PICO}$.

La TOXEMIA se presentó en el $13.8 \%$ del Grupo A y en el $9.3 \%$ del Grupo B. La menor frecuencia la encontramos en el Grupo B, el de mayor número de casadas y de mejor consulta prenatal. La frecuencia de Toxemia en el total de adolescentes estudiadas, $11.5 \%$, es alta si la comparamos con el promedio general del Servicio que oscila en el $6.7 \%$, recordando que su incidencia es muy baja entre nosotros por motivos que aún desconocemos. Los primeros síntomas de Toxemia los encontramos de manera tardía en todas nuestras pacientes, siempre después de la semana 33.

El no haber observado Toxemia en el reducido número de pacientes de 11 y 12 años lo explicamos por cuan-

\section{CUADRO N: 6}

TOXEMIA

\begin{tabular}{|c|c|c|c|c|c|c|c|}
\hline \multirow[b]{2}{*}{ Edad } & \multirow[b]{2}{*}{ pacientes } & \multicolumn{2}{|c|}{ Preeclampsia } & \multicolumn{2}{|c|}{ Eclampsia } & \multicolumn{2}{|c|}{ Total } \\
\hline & & $\mathbf{N}^{0}$ & $\%$ & $\mathrm{~N}^{0}$ & $\%$ & $N^{o}$ & $\%$ \\
\hline Grupo A & 588 & 45 & 7.6 & 31 & 5.2 & 76 & 13.8 \\
\hline Grupo B & 608 & 39 & 6.4 & 18 & 2.9 & 57 & 9.3 \\
\hline TOTAL & 1196 & 84 & 7.0 & 49 & 4.1 & 133 & 11.5 \\
\hline
\end{tabular}

CUADRO N: 7

TOXEMIA EN RELACION CON LA EDAD

\begin{tabular}{|c|c|c|c|c|c|c|c|}
\hline \multirow[b]{2}{*}{ Edad } & \multirow[b]{2}{*}{ pacientes } & \multicolumn{2}{|c|}{ Preeclampsia } & \multicolumn{2}{|c|}{ Eclampsia } & \multicolumn{2}{|c|}{ Total } \\
\hline & & $N^{?}$ & $\%$ & No & $\%$ & $\mathrm{~N}^{\mathrm{o}}$ & $\%$ \\
\hline 11 & - & - & - & - & - & - & - \\
\hline 12 & 1 & - & - & - & - & - & - \\
\hline 13 & 9 & 4 & 44.4 & 3 & 33.3 & 7 & 77.7 \\
\hline 14 & 114 & 19 & 16.6 & 5 & 4.3 & 24 & 20.9 \\
\hline 15 & 464 & 22 & 4.7 & 23 & 4.9 & 45 & 9.2 \\
\hline 16 & 608 & 39 & 6.4 & 18 & 2.9 & 57 & 9.3 \\
\hline TOTAL & 1196 & 84 & 7.0 & 49 & 4.1 & 133 & 11.5 \\
\hline
\end{tabular}


to cuatro de ellas terminaron su gestación en aborto antes de las doce semanas. La mayor incidencia corresponde a las de menor edad, 13 años, $77.7 \%$ y disminuye rápidamente a medida que aumenta la edad, como podemos ver en el Cuadro 7 al estudiar las 1.196 adolescentes que terminaron su gestación en parto.

En los dos Grupos fue sensiblemente igual la incidencia de otras COMPLICACIONES DEL EMBARAZO tales como anemia (con hemoglobina inferior a $10 \mathrm{mg}$.) en el $28.7 \%$, infección de vías urinarias (con urocultivo positivo y más de 100.000 colonias del germen causal) en el $1.2 \%$, sífilis en dos pacientes de 16 años $0.15 \%$, paludismo (malariae y falciparum) en 6 pacientes $0.45 \%$, poliparasitismo intestinal en el $82 \%$, condilomatosis vulvar y vaginal en cuatro $0.3 \%$, problemas de siquismo en dos, $0.15 \%$. Estas complicaciones no son inherentes a la edad sino al medio en que vivimos, comunes a todos los grupos de edad. Sabemos que serias complicaciones durante el embarazo retardan el crecimiento endouterino fetal y por ende el peso de los productos.

Encontramos tres pacientes del Grupo B con EMBARAZO GEMELAR: uno por cada 222 embarazos; el índice del Servicio es de uno por cada 95 embarazos. Estos embarazos terminaron en parto prematuro entre las semanas 26 y 29 , con productos que oscilaron entre los 740 y los 930 gramos con cuatro mortinatos y dos nacidos vivos que murieron en las dos primeras horas de vida.

En relación con la PARIDAD el $98.7 \%$ del Grupo A y el $93.9 \%$ del Grupo B fueron primíparas (Cuadro $8)$.

CUADRO N: 8

GESTACION Y PARIDAD

\begin{tabular}{|c|c|c|c|c|c|c|c|}
\hline \multirow[b]{2}{*}{ Gesta y } & \multirow[b]{2}{*}{ Para } & \multicolumn{2}{|c|}{ Grupo A } & \multicolumn{2}{|c|}{ Grupo B } & \multicolumn{2}{|c|}{ Total } \\
\hline & & No & $\%$ & $\mathbf{N}^{0}$ & $\%$ & $\mathrm{~N}^{\mathrm{e}}$ & $\%$ \\
\hline G. I & Primíparas & 533 & 90.6 & 491 & 80.6 & 1024 & 85.6 \\
\hline \multirow[t]{2}{*}{ G. II } & Primíparas & 37 & 6.3 & 46 & 7.5 & 83 & 6.9 \\
\hline & Secundíparas & 3 & 0.5 & 52 & 8.6 & 55 & 4.5 \\
\hline \multirow[t]{2}{*}{ G. III } & Primíparas & 8 & 1.3 & 3 & 0.4 & 11 & 0.9 \\
\hline & Secundíparas & 2 & 0.4 & 12 & 1.9 & 14 & 1.1 \\
\hline \multirow[t]{2}{*}{ G. IV } & Primíparas & 3 & 0.5 & 2 & 0.5 & 5 & 0.5 \\
\hline & Secundíparas & 2 & 0.4 & 2 & 0.5 & 4 & 0.5 \\
\hline \multicolumn{2}{|c|}{ TOTAL } & 588 & & 608 & & 1196 & \\
\hline
\end{tabular}

De los 1.196 PARTOS (588 del Grupo A y 608 del Grupo B), el $74.8 \%$ fueron espontáneos, el $9.5 \%$ fueron intervenidos con fórceps, el $9.3 \%$ con Vacuum Extractor y el $6.4 \%$ con operación cesárea. Los índices del Servicio para gestantes de mayor edad son: $12.3 \%$ para el fórceps, $9.5 \%$ para el Vacuum y $9.4 \%$ para la cesárea. El fórceps y el Vacuum los empleamos de manera preferencial para acelerar el expulsivo y, en un número muy reducido, para tratar distocias de partes blandas. Las indicaciones y contraindicaciones de las maniobras obstétricas son las mismas para adolescentes y para multíparas de mayor edad (Cuadro 9).

Las PRESENTACIONES muestran índices sensiblemente iguales en los dos grupos, como también en rela- 
CUADRO NN 9

TERMINACION DEL PARTO

\begin{tabular}{|c|c|c|c|c|c|c|}
\hline \multirow[b]{2}{*}{ Parto } & \multicolumn{2}{|c|}{ Grupo A } & \multicolumn{2}{|c|}{ Grupo B } & \multicolumn{2}{|c|}{ Total } \\
\hline & $N^{0}$ & $\%$ & No & $\%$ & $\mathbf{N}^{0}$ & $\%$ \\
\hline Espontáneo & 465 & 78.5 & 428 & 71.1 & 893 & 74.8 \\
\hline Fórceps & 53 & 9.0 & 65 & 10.0 & 118 & 9.5 \\
\hline Vacuum & 39 & 6.6 & 73 & 12.0 & 112 & 9.3 \\
\hline Cesárea & 31 & 5.9 & 42 & 6.9 & 73 & 6.4 \\
\hline TOTAL & 588 & & 608 & & 1196 & \\
\hline
\end{tabular}

CUADRO NN 10

PRESENTACIONES

\begin{tabular}{|c|c|c|c|c|c|c|}
\hline \multirow[b]{2}{*}{ Presentación } & \multicolumn{2}{|c|}{ Grupo A } & \multicolumn{2}{|c|}{ Grupo B } & \multicolumn{2}{|c|}{ Total } \\
\hline & $N^{0}$ & $\%$ & $\mathbf{N}^{0}$ & $\%$ & $\mathbf{N}^{0}$ & $\%$ \\
\hline Cefálica & 534 & 95.8 & 584 & 96.2 & 1148 & 96.0 \\
\hline Pelvis & 13 & 2.3 & 17 & 2.8 & 30 & 2.6 \\
\hline Hombro & - & - & 3 & 0.4 & 3 & 0.2 \\
\hline No precisada & 11 & 1.9 & 4 & 0.6 & 15 & 1.2 \\
\hline TOTAL & 558 & & 608 & & 1196 & \\
\hline
\end{tabular}

ción con los generales del Servicio (Cuadro 10).

Ciento once partos del Grupo A $(18.8 \%)$, y treinta y dos del Grupo B $(5.2 \%)$ fueron PARTOS PREMATUROS, lo que nos da una incidencia de $12 \%$ para adolescentes. El $78.1 \%$ de los partos prematuros se presentó en gestantes solteras. El menor índice de partos prematuros pertenece al Grupo $B$, el de mayor número de casadas, de mayor edad y de mejor asistencia prenatal. (Cuadro 11).

\section{CUADRO NN 11}

PARTO

\begin{tabular}{lrrrrr}
\hline Edad & $\begin{array}{r}\text { Total } \\
\text { Partos }\end{array}$ & \multicolumn{2}{c}{ Prematuro } & \multicolumn{2}{c}{ A término } \\
$\mathbf{N}^{\mathbf{0}}$ & $\%$ & $\mathbf{N}^{\mathbf{9}}$ & $\%$ \\
\hline Grupo A & 588 & 111 & 18.8 & 477 & 81.2 \\
Grupo B & 608 & 32 & 5.2 & 576 & 94.8 \\
TOTAL & 1196 & 143 & 12.0 & 1053 & 88.0 \\
\hline
\end{tabular}

Algunas COMPLICACIONES OBSTETRICAS tales como la desproporción céfalo pélvica, la mola hidatiforme, la placenta previa, el desprendimiento

\section{CUADRO № 12}

\section{COMPLICACIONES OBSTETRICAS}

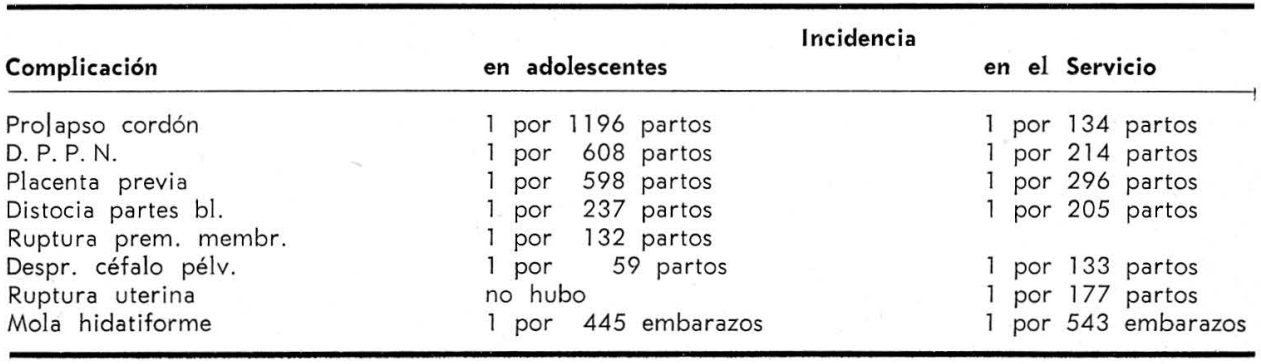


prematuro de placenta normoinserta, el prolapso del cordón, la ruptura uterina, cuando se presentaron, tuvieron un índice inferior al general del Servicio (Cuadro 12).

La DURACION DEL TRABAJO DE PARTO fue igual en los dos grupos: 12 horas en promedio, cifra semejante a la de un grupo de mil primíparas de 20 a 30 años: 11 horas. El trabajo más corto, 4 horas, se encontró en 5 pacientes de 13 y 14 años. Todos los trabajos superiores a 18 horas ( 32 del Grupo A y 24 del Grupo $B$ ) se encontraron en pacientes que llegaron tardíamente al Servicio.

La MORBILIDAD MATERNA durante el puerperio fué:

\begin{tabular}{lrl}
\hline endometritis & 37 & $3.1 \%$ \\
anemia & 21 & 1.8 \\
inercia uterina & 13 & 1.1 \\
dehiscencia de episiorrafia & 10 & 0.8 \\
dehiscencia de pared & 8 & 0.6 \\
mastitis & 6 & 0.4 \\
insuficiencia renal aguda & 2 & 0.1 \\
sicosis puerperal & 2 & 0.1 \\
& -19 & \\
TOTAL & 99 & $8.0 \%$ \\
\hline
\end{tabular}

El Promedio de PERMANENCIA EN EL SERVICIO es de 2.6 días paciente para el parto vaginal y 6.9 para el parto por cesárea, tanto en el grupo de adolescentes como en el de mayor edad.

Tuvimos cuatro MUERTES MATERNAS por eclampsia, todas primíparas solteras: dos del Grupo A $(0.3 \%)$ y dos del Grupo B (0.3\%); una de 13 años murió media hora después de su ingreso, una de quince años murió al tercer día y dos de 16 años (una murió a las 24 horas y otra a los 10 días, ambas por insuficiencia renal aguda). Todas llegaron en estado convulsivo.

En relación con el PESO DE LOS PRODUCTOS encontramos una gran diferencia entre el Grupo A y el Grupo B. E nel Grupo A el 22.3\% tuvo un peso inferior a los $2.500 \mathrm{gr}$., en cambio en el Grupo B sólamente el $11.3 \%$ no alcanzó este peso; ello nus da un índice promedio de prematurez para adolescentes de $16.8 \%$, índice muy alto al compararlo con el 8.1 del Servicio. El $56.3 \%$ del total de adolescentes ( $50.4 \%$ del Grupo A y $62.3 \%$ del Grupo B) dieron a luz. productos con peso superior a los 3.000 gramos. La mayoría de los productos con peso inferior a los 2.500 gr. se encontraron en las pacientes que habían presentado serias complicaciones durante el embarazo (el $39.2 \%$ nacieron de madres con toxemia), dato digno de tenerse en cuenta para futuros trabajos prospectivos. Bien sabemos que el bajo peso del niño al nacer aumenta los riesgos de morbi mortalidad post-natal y que en productos por debajo de 2.000 gr. la mortalidad neonatal aumenta del 4 al 50\%. (Cuadro 13).

Encontramos 25 MORTINATOS en el Grupo A (3.7\%), y 21 en el Grupo B $(3.1 \%)$; ello representa una MOR-

CUADRO NN 13

PESO DE LOS PRODUCTOS

\begin{tabular}{|c|c|c|c|c|c|c|}
\hline \multirow[b]{2}{*}{ Peso de los productos } & \multicolumn{2}{|c|}{ Grupo A } & \multicolumn{2}{|c|}{ Grupo B } & \multicolumn{2}{|c|}{ Total } \\
\hline & $\mathbf{N}^{0}$ & $\%$ & $\mathbf{N}^{0}$ & $\%$ & $\mathbf{N}^{\mathbf{o}}$ & $\%$ \\
\hline menos de $2.500 \mathrm{~g}$. & 131 & 22.3 & 69 & 11.3 & 200 & 16.8 \\
\hline 2.500 a $2.999 \mathrm{~g}$. & 156 & 26.5 & 149 & 24.5 & 305 & 25.5 \\
\hline 3.000 a $4.460 \mathrm{~g}$. & 297 & 50.4 & 378 & 62.3 & 275 & 56.3 \\
\hline no especificado & 4 & 0.8 & 12 & 1.9 & 16 & 1.4 \\
\hline
\end{tabular}


TINATALIDAD para productos de adolescentes de $3.4 \%$, inferior a la general del Servicio que es de $5.4 \%$.

Veintiún MORTINEONATOS eran del Grupo A $(3.3 \%)$ y 23 del Grupo $\mathrm{B}(3.5 \%)$, correspondiendo en su mayoría a productos inmaduros, a hijos de pacientes con eclampsia o con amniotitis $y$, en algunos, con componente iatrogénico.

EI INDICE DE MORTALIDAD PERINATAL no depurada es de $6.8 \%$ para el total de adolescentes, índice inferior al general del Servicio que es de $7.18 \%$.

LaS MALFORMACIONES CONGENITAS fueron aparentes en 7 productos del Grupo A y en 5 del Grupo B $(1.2 \%$ y $0.8 \%$ respectivamente), lo que nos dá un índice de $1 \%$ para el total de adolescentes, cifra similar a la del grupo de mayor edad que es de $0.97 \%$.

\section{CUADRO № 14}

\section{MALFORMACIONES CONGENITAS}

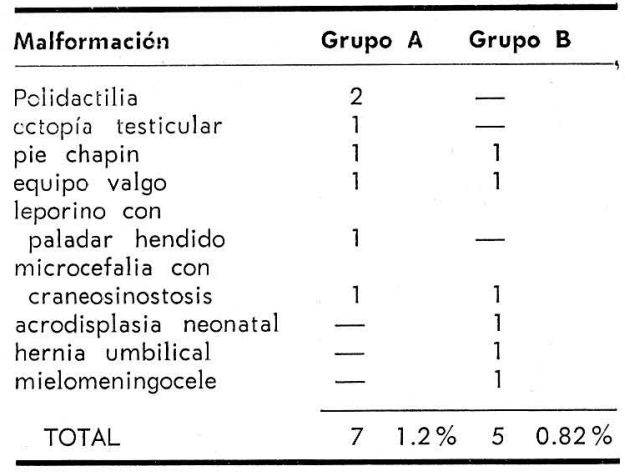

\section{Comentarios}

El descenso de gestantes adolescentes a partir de 1972 puede obedecer a la campaña de planificación familiar adelantada en el Servicio Hospitalario, en los diferentes Centros de salud y en Profamilia, como también a los ciclos de orientación sexual que en gran escala se están dando a conglomerados en los barrios de la ciudad. Si a esta campaña agregáramos la cátedra de orientación sexual en escuelas y colegios desde temprana edad como lo prevee el pensu moficial para educación primaria en Colombia, veríamos, ciertamente, una baja muy apreciable del número de embarazos en el despertar de la vida.

Este comienzo temprano de orientación sexual está justificado en el Cuadro 2 donde apreciamos que la menarca en nuestro medio se presenta a muy corta edad y que las relaciones sexuales no son desconocidas por muchas niñas hasta de 10 años.

La falta de asistencia al control prenatal, común en todos los estudios revisados $(14,16,22,28,30)$ se explica por múltiples razones, siendo tal vez la principal el hecho de que el $62.5 \%$ sean solteras. La vergüenza a que se descubran sus relaciones sexuales prematrimoniales, 0 su embarazo oculto, o la decisión a desconocer su situación, o la ignorancia, o la rebeldía, las alejan del control prenatal.

Las gestantes solteras de esta edad son personas sin hog ar formado en el $85 \%$ de los casos, repudiadas muchas veces por los padres y familiares, "expulsadas" de escuelas y colegios por su actitud, miradas con recelo par la sociedad, que van a la deriva sin profesión alguna definida, carentes de toda preparación para trabajo alguno, expuestas a la prostitución o a la vagancia. Ello hace que a más del trauma emocional agreguen la falta de techo, la alimentación insuficiente, al dieta voluntaria, más compresión abdominal con fajas para ocultar la gestación, la falta de dirección y orientación hacia un cen- 
tro de control prenatal, como resultado de condiciones culturales, legales, sociales y emocionales, todas de difícil pero de posible solución; factores éstos que aumentan la incidencia de anemia, de desnutrición, de enfermedades venéreas y que elevan el índice de abortos, de toxemia, de partos prematuros, de productos de bajo peso, de malformaciones congénitas y demás complicaciones obstétricas no inherentes al factor edad, sin la menor duda. Prueba de ello el hecho de que en el Grupo B, con mayor porcentaje de casadas y por ende con mejor estabilidad emocional y socio económica, bajan considerablemente las tasas de complicaciones obstétricas y de sus secuelas.

Se admite que el factor etiológico primordial del aborto en gestantes muy jóvenes es la baja edad; sin embargo, nadie ha investigado las anomalías uterinas, las zoonosis, las hemopatías y una serie de alteraciones funcionales que hayan podido acarrear el aborto de las pacientes estudiadas. La observación de que cuando la adolescente aumenta en edad y adquiere una cierta estabilidad socioeconómica merced al matrimonio, disminuye el índice de abortos, $f$ de que éstos se presentan con mayor frecuencia en el grupo de solteras, nos autoriza a pensar que un factor principal etiológico podría ser el factor síquico, emocional, y no el factor edad.

Nuestra casuística, como todas las consultadas $(2,5,6,7,9,10,11,14$, $17,18,20,22,25,28,29$ ) dan un mayor índice de Toxemias en las adolescentes de menor edad $(28 \%$ en la serie de Musio para adolescentes de 12 a 14 años). Clark afirma que la toxemia es el mayor problema en las adolescentes muy jóvenes (9) tal vez por mala disposición en el desarrollo para la reproducción o por falta de madurez del sistema endocrino (20,
24). Schmitz y Towe dicen que fuera de la gran incidencia de toxemia no tuvieron ctro problema obstétrico ni en el embarazo, ni en el parto ni en el puerperio de doscientas gestantes adolescentes (29). Algunos autores observan en sus series que a pesar de los buneos cuidados prenatales, las adolescentes grávidas tienen una mayor incidencia de toxemia que las mujeres de mayor edad (9, 20, 29). La sintomatología toxémica en nuestras pacientes fue más benigna que en las del grupo de mayor edad, y regresaron más rápidamente a la normalidad, salvo en las cuatro pacientes que murieron. En ninguna de las otxémicas encontramos alsa tensional por encima de 18; en cambio la proteinuria se manifestó con cifras elevadas en un gran procentaje. No encontramos aumento exagerado de peso. Moreno y colaboradores observaron (22) que las eclámpticas mayores de 17 años acusaron prematuridad y morbilidad neonatal más elevada que las de menor edad.

Será el factor edad por sí mismo el causante del alto índice de toxemia? Es una pregunta que está en estudio y cuya respuesta aún desconocemos. Es un enigma. Hay múltiples causas en estas pequeñas pacientes que bien podrían discutirse como predisponentes: constitucionales o somáticas unas, tales como la desnutrición crónica, la anemia, la hipoarterialización primaria del útero $(3,15$, 22); comunes otras a toda gestante, tales como las infecciones urinarias, el embarazo molar, el embarago gemelar, el polihidramnios, la sobredistensión uterina; y primordialmente las de origen síquico por el tremendo impacto emocional que las lleva rápidamente a la anorexia, al insomnio, a la hiperemesis, a un estado de permanente zozobra, a la desesperación. 
En el $37.8 \%$ de las pacientes a quienes se les practicó operación cesárea encontramos como causa la desproporción feto pélvica; como dato curioso, todos los productos en estos casos estuvieron por debajo de los 3.000 gr., en tanto que en partos con productos de mayor peso, que lo fueron el $56.3 \%$ no encontramos este factor causal, lo que nos hace pensar que pudiera haber sido una "manera paternal", muy explicable por cierto, de acortar en estas niñas el tiempo de trabajo de parto, sobre todo en aquellas que llegaron al Servicio con trabajo prolongado. En ninguna se hizo diagnóstico radiológico de estrechez pélvica. Bien sabemos que la porción ósea del conducto pélvico es adecuada para el paso de un producto normal a término pocos meses después de la menarca. La menor edad no implica un aumento en el índice de cesáreas $(7,12,25)$; ello hizo decir a Harris en 1922 que la época ideal de una mujer para tener hijos está entre los 12 y los 16 años (17).

El índice de productos de bajo peso al nacer, $16.8 \%$, no lo consideramos alto si tenemos en cuenta que el $12 \%$ de los partos fue prematuro $y$ que en ellos jugaron papel preferencial complicaciones importantes tales como la desnutrición, la anemia, el paludismo, la toxemia, el desprendimiento prematuro de placenta normoinserta. Esto nos induce a pensar que el factor edad por sí no es el único causante del bajo peso de los productos.

Se ha dicho que hay un mayor índice de deficiencia mental en hijos de madres menores de 15 años. Los cuatro casos que hemos podido constatar corresponden a productos de madres eclámpticas nacidos con peso inferior a los 2.000 gramos. "Los estudios de predicción y retrospectivos concuerdan, en general, en que el nacido prematuramente se halla en desventaja manifiesta en cuanto a los aspectos de complicaciones neurológicas y deficiencia mental" y se ha encontrado alta frecuencia de prematurez entre los escolares retrasados (1, 3).

La mortinatalidad, $3.1 \%$ encuentra explicación en el índice de eclampsia y en la presencia de otras severas complicaciones del embarazo y del parto, causantes siempre de una tasa muy elevada de mortinatalidad (1, 23, 32 ).

La mortalidad neonatal temprana, $3.4 \%$, es la respuesta lógica al bajo peso de los productos al nacer y a la patología que encontramos en nuestras pacientes durante su gestación o su parto, complicaciones no inherentes a la adolescente: sífilis, paludismo, epilepsia, anemia, desnutrición, DPPN, ruptura prematura de membranas, trabajo prolongado, a más de las malformaciones congénitas mayores y de tantas otras que se nos pasan desapercibidas aún post mortem por falta de autopsia. Ello nos permite pensar que la edad no sea el factor primordial determinante del aumento de mortalidad perinatal ni de la morbilidad de estos productos (15). El índice de mortalidad perinatal en nuestra casuística es inferior al General del Servicio.

Está demostrado que hay una fuerte relación entre la mortalidad perinatal y la clase económica, y que la prematurez y sus complicaciones son los factores causales primarios o de asociación que guardan relación con la mortalidad perinatal (26).

\section{Conclusiones}

La adolescente en gestación puede llevar su embarazo a término, con un producto de peso normal, sano, al 
igual que las gestantes de mayor edad y del mismo nivel socio económico.

El verdadero problema del embarazo a esta temprana edad es la Toxemia, causante de los partos prematuros, de los productos de bajo peso al nacer, de la morbimortalidad materno fetal $y$, por ende, del grave peligro del retardo mental de los productos.

Las complicaciones del embarazo, del parto y del puerperio se encuentran con una incidencia muy similar a la observada en grupos de mayor edad y de iguales condiciones socio económicas. El factor edad en sí, no es el responsable directo de tales complicaciones.

La indicación y contraindicación de las maniobras obstétricas en nada se diferencian de las conocidas para la generalidad de las pacientes.

Teniendo en cuenta el alto, imprevisible e incontrolable índice de toxemias, el tremendo impacto emocional causante de serias complicaciones durante el embarazo, el parto y el puerperio, y las funestas repercusiones socioeconómicas que conlleva el embarazo en adolescentes, nos obliga a considerarlo siempre como "EMBARAZO DE ALTO RIESGO".

La profilaxis sería la formación moral de la niña en el hogar, la orientación sexual desde temprana edad y más tarde el control natal, cuando se haga necesario.

\section{Resumen}

Al estudiar 1.335 embarazos en adolescentes hasta de 16 años encontramos una incidencia de un embarazo por cada 66 pacientes del Servicio. El $10.35 \%$ terminaron en aborto. La Toxemia se presentó en el $11.5 \%$. El $74.8 \%$ de los partos fueron espontáneos, 9.5\% por fórceps, 9.3\% por vacuum y $6.4 \%$ por operación cesárea. El $12 \%$ de los partos fueron prematuros. Las complicaciones obstétricas fueron inferiores a las del grupo de mayor edad. La duración del trabajo de parto fue de 12 horas. Tuvimos mortalidad materna de $0.3 \%$ en eclámpjticas. El $16.8 \%$ de los productos tuvieron un peso inferior a los 2.500 gramos. El $3.4 \%$ de los productos fueron mortinatos y el $3.4 \%$ mortineonatos. En el $1 \%$ de los recién nacidos hubo malformaciones congénitas aparentes. Se dan conclusiones.

\section{BIBLIOGRAFIA}

1 APGAR, V. Comentarios de la prematurez. Clin. Obstét. Gin. de Norte Am. 741, 1964.

2 AZNAR, R. and BENNET, A. E. Pregnancy in the adolescent girl. Am. J. Obst. Gyn. 81 : 934, 1961.

3 BALLARD, W. M. y GOLD, E. M. Aspectos médicos y de salud de la reproducción en la adolescencia. Clín. Obstét. Gin. Norte Am. 338, 1971 .

4 BRIGS, R. M., MARREN, R. R. and THOMPSON, W. B. Pregnancy in the young adolescent. Am. J. Obstet. Gynec. 84: 436. 1962.

5 CASTELLANOS, R., AGUERO, O., FRANCO, G. Y MORENO, L. Estudio sobre mil madres adolescentes menores de 15 años. Rev. Obst. Gin. Venezuela. 29: 65, 1969.

6 CASTELLANOS, R., AGUERO, O., FRANCO, G. y ALLER, J. Estudio de la gestante adolescente. Rev. Obst. Gin. Venezuela. 30 : 197, 1970.

7 CASTELLANOS, R., AGUERO, O., FRANCO G. y ALLER, J. Cesárea en adolescente precoz. Rev. Obst. Gin. Venezuela. 31: 439, 1971.

8 CASTELLANOS, R., AGUERO, O., ALLER, J., FRANCO, G. y NEUMAN, G. Problemas de la gestante de 15 y 16 años de edad. Rev. Obst. Gin. Venezuela. 32: 2, 1972.

9 CLARK, J. F. Toxemia is major complication in teen pregnancy. Ob. Gyn. News. 5: 35, 1970.

10 CLARK, J. F. Obstetricia en adolescentes. Deducciones obstétricas y ginecológicas. Clin. Obstét. Gin. Norte Am. 1026, 1971. 
11 CODEMUS, M. y FANEITE, P. Gestaciones juveniles. Rev. Obst. Gin. Venezuela. 31: 427, 1971.

12 DUARTE CONTRERAS, A. Embarazo en adolescentes de 11 a 15 años. Rev. Colomb. Obst. Gin. 24: 259, 1973.

13 FERNANDEZ, J. A. Mesa redonda sobre embarazo de alto riesgo. Memorias VII Congr. Lat. Amer. Obst. Gin. Quito. Tomo 3 No $3515,1973$.

14 GOMEZ, P. J. M. Obstetricia en adolescentes. Rev. Colomb. Obst. Gin. 23: 353, 1972.

15 GUERRERO, S. A., CHAVEZ, A. J., GONZALEZ, H. R., PEREDO, E. J. J. Y KARCHMER, K. $S$. El riesgo del embarazo en la paciente adolescente. Ginec. Obstet. Méx. 221: 119, 1975.

16 HASSAN, H. M. and FALLS, F. H. The young primipara. Am. J. Obstet. Gynec. 88 : $256,1964$.

17 HUFFMAN, J. W. "Embarazo en las jóvenes y adolescentes" en Ginecología en la infancia y en la adolescencia. Ed. Salvat. Pág. $589,1971$.

18 ISRAEL, S. L. and WONTERSZ, T. B. Teenage obstetrics. Am. J. Obstet. Gyn. 85: 659, 1963.

19 LEWIS, B. V. and NASH, P. J. Pregnancy in patients under 16 years. Brit. Med. J. 2: $733,1967$.

20 MARCHETTI, A. and MENAKER, J. Pregnancy and the adolescent. Ann. J. Obst. Gynec. 59: $1013,1950$.

21 MORALES, P., ARCAY, A. y ECCARI, R. Primiparidad precoz. Rev. Obst. Gin. Venezuela. $30: 225,1970$.
22 MORENO, L. F., MORENO, E. M. y SANABRIA, D. B. Preeclampsia y eclampsia en la adolescente muy joven. Rev. Obst. Gin. Venezuela. 25: 147, 1975.

23 MORENO, L. F. Y MORENO, E. M. Morbiletalidad perinatal en eclampsia. Rev. Obst. Gin. Venezuela. 34: 369, 1974.

24 MONTAGU, A. The reproductive development of the female. 2a. ed. The Julian Press, Inc. New York, 1957.

25 MUSSIO, T. Primigravidas under age 14. Am. J. Obst. Gyn. 84: 442, 1962.

26 OSOFSKY, N. J. y KENDAL, N. Pobreza como factor de riesgo. Clin. Obstét. Gin. Norte Am. 101, 1973.

27 PCLIAKOFF, S. R. Pregnancy in the young primigravida. Am. J. Obstet. Gynec. 76: 746, 1958.

28 RUIZ, Y. V. y PERAZA, Z. M. A. Gestación en la adolescente. Ginec. Obstet. Méx. 208: $117,1974$.

29 SCHMITZ, M. and TOWNE, J. Adolescent primigravida. Surg. Gynec. and Obst. 84 : 962. 1947.

30 SUAREZ, D., LAGUNA, E., FARIA, N. y NEUMAN, G. Nuestra gestante joven. Rev. Obst. Gin. Venezuela. 30: 205, 1970.

31 SZCZEDRIN, W. Estudio de la embarazada adolescente. Rev. Obst. Gin. Venezuela. 35: 25, 1975.

32 WALLACE, H. M. Factores relacionados con la mortalidad y morbilidad perinatales. Clin. Obstét. Gin. Norte Am. 13, 1970. 